\title{
Column Store for GWAC: A High-cadence, High-density, Large-scale Astronomical Light Curve Pipeline and Distributed Shared-nothing Database
}

\author{
Meng Wan ${ }^{1,2,3,4}$, Chao Wu $u^{1,4,7}$, Jing Wang ${ }^{1,4}$, Yulei Qiu ${ }^{1,4}$, Liping Xin ${ }^{1,4}$, Sjoerd Mullender ${ }^{3}$, Hannes Mühleisen ${ }^{3}$, Bart Scheers ${ }^{3}$, \\ Ying Zhang ${ }^{3,5}$, Niels $\mathrm{Nes}^{3}$, Martin Kersten ${ }^{3,5}$, Yongpan Huang ${ }^{6}$, Jinsong Deng ${ }^{1,4}$, and Jianyan Wei ${ }^{1,4}$ \\ ${ }^{1}$ National Astronomical Observatories, Chinese Academy of Science, 20A Datun Road, Chaoyang District, Beijing 100012, China; cwu@nao.cas.cn \\ ${ }^{3}$ University of Chinese Academy of Sciences, Beijing 100049, China
${ }^{2}$ entrum Wiskunde \& Informatica, 1098 XG Amsterdam, The Netherlands \\ ${ }^{4}$ Key Laboratory of Space Astronomy and Technology, National Astronomical Observatories, Chinese Academy of Sciences, Beijing 100012, China \\ ${ }^{5}$ MonetDB Solutions, 1098 XG Amsterdam, The Netherlands \\ ${ }^{6}$ College of Economics, Capital University of Economics and Business, Beijing 100070, China \\ Received 2015 October 22; accepted 2016 April 18; published 2016 September 19
}

\begin{abstract}
The ground-based wide-angle camera array (GWAC), a part of the SVOM space mission, will search for various types of optical transients by continuously imaging a field of view (FOV) of 5000 degrees $^{2}$ every $15 \mathrm{~s}$. Each exposure consists of $36 \times 4 \mathrm{k} \times 4 \mathrm{k}$ pixels, typically resulting in $36 \times \sim 175,600$ extracted sources. For a modern time-domain astronomy project like GWAC, which produces massive amounts of data with a high cadence, it is challenging to search for short timescale transients in both real-time and archived data, and to build long-term light curves for variable sources. Here, we develop a high-cadence, high-density light curve pipeline (HCHDLP) to process the GWAC data in real-time, and design a distributed shared-nothing database to manage the massive amount of archived data which will be used to generate a source catalog with more than 100 billion records during 10 years of operation. First, we develop HCHDLP based on the column-store DBMS of MonetDB, taking advantage of MonetDB's high performance when applied to massive data processing. To realize the real-time functionality of HCHDLP, we optimize the pipeline in its source association function, including both time and space complexity from outside the database (SQL semantic) and inside (RANGE-JOIN implementation), as well as in its strategy of building complex light curves. The optimized source association function is accelerated by three orders of magnitude. Second, we build a distributed database using a two-level time partitioning strategy via the MERGE TABLE and REMOTE TABLE technology of MonetDB. Intensive tests validate that our database architecture is able to achieve both linear scalability in response time and concurrent access by multiple users. In summary, our studies provide guidance for a solution to GWAC in real-time data processing and management of massive data.
\end{abstract}

Key words: astronomical databases: miscellaneous - catalogs

Online material: color figures

\section{Introduction}

\subsection{GWAC Scientific Goals and Data Challenges}

The ground-based wide-angle camera array (GWAC) is a set of ground-based instruments under the framework of the SVOM mission. SVOM is a Chinese-French space mission dedicated to detecting gamma-ray bursts (GRBs) which has been funded by the China National Space Administration and the Centre National d'Etudes Spatiales and is planned to launch in 2021 (Cordier et al. 2015). GWAC is designed to comprise 36 cameras, each with an $18 \mathrm{~cm}$ diameter and $12.8 \times 12.8$ degrees $^{2}$ field of view (FOV). The 36 cameras will point to the sky in different directions and totally cover an area of more

\footnotetext{
${ }^{7}$ Author to whom any correspondence should be addressed.
}

than $5000 \mathrm{deg}^{2}$. Each camera will take an image once every 15 $\mathrm{s}(10 \mathrm{~s}$ exposure plus $5 \mathrm{~s}$ readout). GWAC will simultaneously monitor an area of sky within the FOV of ECLAIRs (Cordier et al. 2015), so that GWAC has the potential to catch the prompt optical emission of GRBs. Besides monitoring GRBs, GWAC is also able to search for other optical transients such as supernovae and the optical counterparts of gravitational-wave bursts.

Thanks to GWAC's 15 s exposure and large FOV, it can also provide light curves with a high time resolution of millions of objects over a long timescale. A light curve is a time series of light intensity representing the magnitude of a celestial object or region as a function of time. In light curve analysis, we are interested in variable objects during periods that show drastic changes. The huge amount of of light curve data will be used 
not only for studying variable stars, but also for searching for transient phenomena such as short timescale gravitational microlensing events and transits by extrasolar planets. It is worth noting that gravitational microlensing events with timescales of less than several hours are unique candidates for searching for interstellar dark objects, for example, freefloating planets. The large amount of data representing the source catalog and light curves produced by GWAC are an important motivation for our data analysis system.

To generate the light curves, the GWAC light curve processing system will face stringent demands on data cadence and the rate of data acquisition of GWAC. One GWAC camera will capture an image every $15 \mathrm{~s}$. The source extraction and subsequent light curve processing of each image should be finished in a time frame of $15 \mathrm{~s}$. This is due to the fact that short timescale observing objects, such as microlensing events, need to be discovered by analyzing light curve data in real-time. The data rate of each camera is $\sim 12,000$ source measurements (2.4 MB) per second, which means that the total data rate is $85 \mathrm{MB} / \mathrm{s}$ for the whole GWAC array system. GWAC will produce $\sim 2.7 \mathrm{~TB}$ catalogs data per day and $\sim 9 \mathrm{~PB}$ over the designed 10 years of operation.

\subsection{Comparison with Other Surveys}

The massive catalog and light curve data produced by GWAC motivate us to use relational databases to realize data manipulation and query, which is a popular solution for similar modern time-domain survey projects.

Distributed relational database systems (RDBMS) are widely used to manage the large-scale observational data produced by large-aperture, wide-field surveys such as Pan-STARRS and LSST. Pan-STARRS (Kaiser et al. 2002; Burgett 2012) is designed to collect data at a rate of $3 \sim 10$ terabytes (TB) per night. The observed data of Pan-STARRS are managed in a distributed relational Microsoft SQL server database, which is spatially partitioned into slice databases using a hash function over the spatial location (RA and DEC) of each detection (Simmhan et al. 2011). The raw imaging data of LSST is expected to be about 15 TB per night (Ivezic et al. 2008). Over the 10 years of LSST survey operations, LSST will result in over 50 PB for catalog databases (Jurić et al. 2015). To manage the massive amount of data in the astronomical catalog, LSST developed a special distributed, shared-nothing SQL database query system, called Qserv, which is independent of a particular RDBMS (Wang et al. 2011; Becla et al. 2013; Becla \& Wang 2014).

In addition to managing the large amount of observational data, the RDBMSs also play an important role in the data processing pipeline of radio transient search projects (Norris 2010), such as VAST (variables and slow transients) of the Australian Square Kilometer Array Pathfinder (Murphy et al. 2013) and LOFAR (the LOw-Frequency ARray; Van
Haarlem et al. 2013). The transient processing of VAST is implemented as a real-time pipeline which employs the PostgreSQL database with the Q3C (Koposov \& Bartunov 2006) plugin to optimize coordinate searches and crossmatches (Banyer et al. 2012). Its source data rate is $\sim 12,000$ source measurements per second. The transient pipeline TraP (Scheers 2009; Swinbank et al. 2015) of LOFAR is implemented in real-time processing and developed on the column-store database MonetDB (Scheers et al. 2012). ${ }^{8}$ Its source data rate is $\sim 10-10,000 / \mathrm{sec}$. Pioneering column-store technology (Abadi et al. 2013) since 1993, MonetDB has achieved significant speed up compared to traditional databases through innovations at all layers of its DBMS (Idreos et al. 2012, 2007; Manegold et al. 2009). In column store, queries only touch the relevant columns, and when in contiguous memory it allows compression and good cache-hit ratios. Furthermore, MonetDB's kernel is a programmable relational algebra machine operating on "array"-like structures, which is exactly what CPUs are good at. Thanks to the highperformance computing capability provided by the MonetDB database, the TraP pipeline has successfully realized transient search and light curve generation in real-time through the technology of the table-driven logic of MonetDB.

The success of LOFAR motivates us to employ MonetDB as the database platform for the GWAC high-cadence, highdensity light curve pipeline (HCHDLP) because the scientific goal and data processing strategy of GWAC are similar to those of LOFAR. This satisfies one of Jim Gray's laws: a large-scale scientific database should bring computations to data, rather than data to computations (Gray et al. 2005). In addition, the latest distributed technology of MonetDB (see our discussion in Section 3) can support a distributed data architecture, which suits our long-term data storage and queries. HCHDLP of GWAC is not based on the heritage of either Qserv of LSST or Pan-STARRS because they are not using database-centric computing approaches for their dynamic pipelines during data production.

The organization of the paper is as follows. Section 2 provides an overview of HCHDLP and th optimization techniques to achieve real-time performance for each camera. In Section 3, we design a shared-nothing distributed database on top of the GWAC camera array. In Section 4, we present the evaluation of the light curve pipeline in terms of functionality and performance. We discuss future research directions in Section 5 .

\section{MonetDB-based HCHDLP}

The following are two of the main goals of the HCHDLP: (1) to manage a catalog that contains all of the individual measurements of the sources observed by GWAC; (2) to

\footnotetext{
8 Centrum Wiskunde and Informatica (CWI), the Netherlands (www. monetdb.org).
} 
GWAC hardware architecture
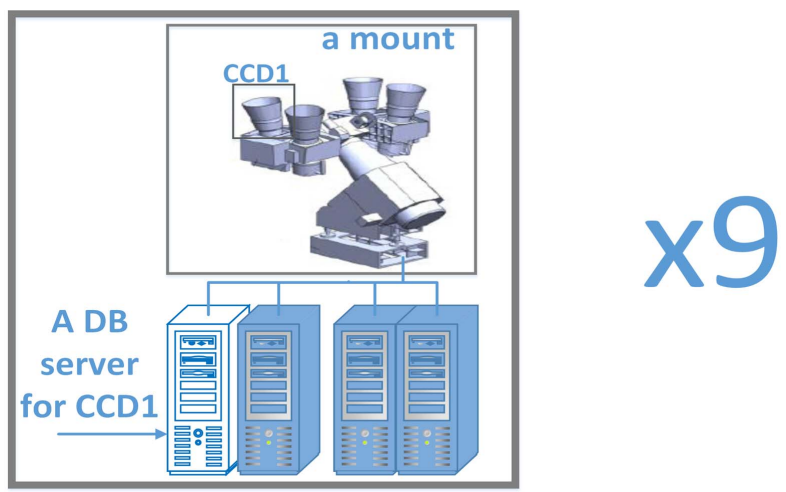

Figure 1. GWAC telescope array and its computing cluster, in which each CCD (telescope) has a dedicated database server, e.g., CCD1 has an accompanying database server displayed in white, and so on. The HCHDLP database is temporary where the bulk of in-database processing takes place to achieve real-time data processing, with only limited disk capacity. The catalog and light curve production of the temporary database will be periodically exported to a long-term archival catalog database. The long-term database is a distributed shared-nothing database illustrated in Figure 6.

(A color version of this figure is available in the online journal.)

create cumulative light curves with which to study variable sources and transients (microlensing events, etc.) in real-time.

We designed the HCHDLP based on the following requirements and constraints of GWAC. First, the total processing time of an image should not exceed the time between two subsequent images, i.e., $15 \mathrm{~s}$. Second, the data rate needed to be processed by HCHDLP is $\sim 12,000$ sources/sec. Finally, the design of HCHDLP should satisfy the boundary conditions given by the scientific requirements and hardware architecture.

The GWAC hardware architecture is illustrated in Figure 1. There are nine mounts in total, and each mount consists of four CCD cameras where each camera uses a dedicated database server. The HCHDLP database is temporary; the long-term database is a distributed shared-nothing database, which is illustrated in Figure 6 in Section 3.

The data flowchart of GWAC is shown in Figure 2. The input to HCHDLP is a stream of catalog data following image preprocessing, quality control, source extraction, and flux calibration of pipeline 0. First, the CCD reductions and astrometry calibration are processed in the image preprocessing. Then, the quality control filters out the bad quality image according to star profiles, star numbers, and astrometry accuracy. After quality control, the images of good quality are taken through the source extraction procedure. Then, the zone ID and Cartesian coordinates are calculated from equatorial coordinates. Finally, the flux calibration is carried out through a comparison to standard stars in the same frame. The standard stars are referenced from the UCAC4 catalog
(Zacharias et al. 2013). The resulting source attributes of the final calibrated catalog are listed in Table 1. All of the sources are in the form of point-like sources and will be loaded into the target table in Figure 3.

The HCHDLP consists of two procedures, i.e., data loading, and source association and light curve creation. In order to load large amounts of data quickly, binary bulk loading is adopted for MonetDB to ingest the point-like source catalogs using a parallel data insertion command. The source association identifies every source detected by GWAC and concatenates all of the current and archived measurements of each identified source in the time series, resulting in the light curves.

Due to the high cadence, a lot of the data processing is shipped to the database engine. Database algorithms take care of source associations. Figure 3 shows the entity relationship diagram of five key tables and their one-to-many relationships.

It is worth noting that the measured coordinates of the sources simultaneously take two forms: two components in the form of spherical coordinates representing R.A. and DEC, and three components of Cartesian coordinates in the form of unit vectors representing $\mathrm{x}, \mathrm{y}, \mathrm{z}$, in order to save time during complicated source association calculations.

In practice, when a new source is loaded into the target table, it is assigned a new permanent ID, ID_target, by a primary key as its unique identifier. Then, the new source is associated with previous records of sources in the uniquecatalog table, in which each source has been assigned an ID_uniq. If the new source is a good match with a record in the uniquecatalog, then the associated IDs of ID_target and ID_uniq are appended into the association table associatedsource, which is a de facto light curve table. If the match failed, then a new record is created in the uniquecatalog. The related auxiliary information is recorded in the table image and skyarea.

An association pair is acceptable if the angular distance between the two sources is smaller than a given tolerance radius. Generally speaking, the choice of the tolerance radius is mainly based on the uncertainty of the astrometry calibration. However, for the GWAC system, due to its large pixel scale of $\sim 12$ arcsecond per pixel, the crosstalk between sources will be a problem when getting the positions of these sources in the CCD image, especially for those objects with a low signal-tonoise ratio (Dudik et al. 2012). In these sub-sampled images, the point-spread function (PSF) of the sources would be relatively stable since it is less sensitive to variations of the environment, like observation or temperature. Thus, in our practical experiments, 2.5 pixels, which is $\sim 1.4$ times the typical PSF value ( 1.8 pixels), is a proper choice for GWAC images when performing the cross-match.

From our analysis, the mismatch rate is $\sim 0.05 \%$ (considering the flux constraint) with our above tolerance radius, while the mismatch rate is $<0.1 \%$ if not considering the flux constraint. This mismatch rate is acceptable for GWAC taking into account the balance with data processing speed. Additionally, 


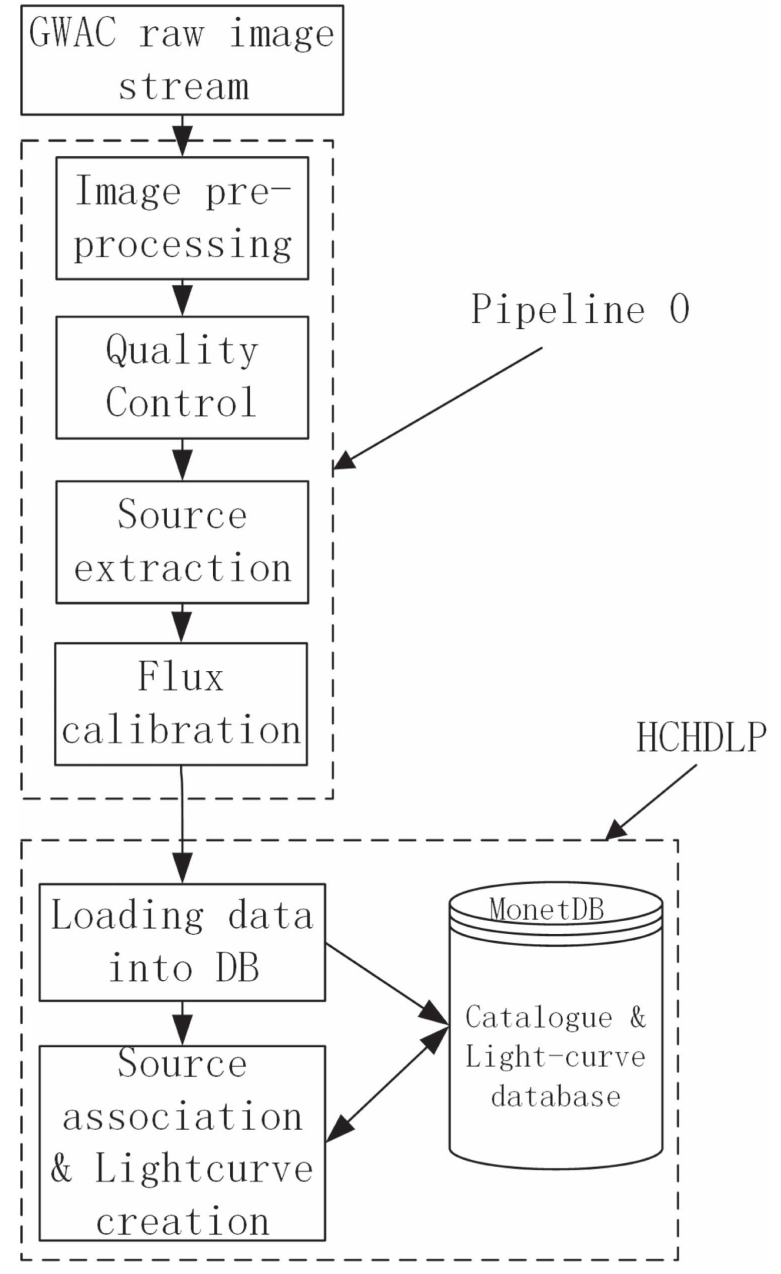

Figure 2. Data flowchart of GWAC. After the preceding "source extraction" module is applied, detections and measurements related to celestial objects in the images are extracted into catalog files. In the last procedure of pipeline 0 , flux calibration is carried out through comparison to standard stars in the same fields. The standard stars are selected from the UCAC4 catalog. Then, in the HCHDLP phase, the catalog files are inserted into the HCHDLP database where they are associated with an existing sky-model uniqcatalog table one by one to form light curves.

the color constraint in the cross-match can be ignored since all of the data used for the association are acquired from the same camera and the same band.

The HCHDLP database uses the popular zone algorithm (Gray et al. 2007) to speed up processing. Its basic idea is to map a sphere into equally spaced declination zones. Using the ZoneID filter, the strength of the zone algorithm comes from its simplicity and the locality it produces: a zone only has two neighbors when matching two data sets. We only need to look for matches within the same ZoneID and its neighbors. The optimal zone height is set to the value of the tolerance radius. Both "tall" and "short" zone heights will cause more neighbors that need to be joined with the center zone. These extra areas add extra costs that outweigh the savings in pair-wise comparisons (Gray et al. 2004). The search radius can be easily set at any time as an input parameter of the association function.

\subsection{Optimization of Source Association}

A straightforward, zone-based source association query that joins two tables using the Euclidean distance would run for a very long time on high-cardinality data sets. Among all of the relational algebra operators in the association, the Join operator is the most expensive. In the Join operation, each zone in the left reference table "uniquecatalog" needs to be compared with all of the zone values in the right "target" table one by one. The zone search results must be further reduced by an $r a$ filter of the Alpha(theta, decl) computation. The inflated radius Alpha function can compute the limiting $r a$ ranges of points in all regions both near the equator and near the poles (see details in Gray et al. 2007). Then, a quick filter on $d e c$ is tested and, finally, a careful Euclidean distance is computed. The time complexity of this operation is $\mathcal{O}\left(n^{2}\right)$. For example, this straightforward query takes several hours to associate two tables each with $\sim 175,600$ tuples. The SQL extract below is the straightforward source association query.



To significantly reduce the large amount of time required for source association, we optimized the association for both time and space complexity by two means: from outside the database (SQL clauses) and inside the databases (query optimizer).

From outside the database engine, from a SQL perspective, since the sources stored in the table target are unordered, this is also true for the zoneid column, which leads to the zoneid lookup being a random access pattern. If the randomly accessed data are too large for the $\mathrm{CPU}$ caches, the random access will cause cache misses and performance degradation (Boncz et al. 1999). For this reason, we create a sorted version of the 
Table 1

Measured Attributes of Each Source

\begin{tabular}{lll}
\hline \hline Name & Type & Description \\
\hline ID & long int & Every inserted source/measurement gets a unique id, generated by the source extraction procedure. \\
imageid & int & The reference ID to the image from which this source was extracted. \\
zone & smallint & The zone ID in which a source declination resides, calculated by the source extraction procedure. \\
ra & double & Right ascension of the source (J2000 degrees), calculated by the source extraction procedure. \\
dec & double & Declination of a source (J2000 degrees). As above. \\
mag & double & The magnitude of a source. \\
mag_err & double & The error of magnitude. \\
pixel_x & double & The instrumental position of a source on CCD along x. \\
pixe_y & double & The instrumental position of a source on CCD along y. \\
ra_err & double & The 1-sigma error on ra (degrees). \\
dec_err & double & The 1-sigma error on declination (degrees). \\
x & double & Cartesian coordinates representation of RA and declination, calculated by the source extractor procedure. \\
y & double & Cartesian coordinates representation of RA and declination, as above. \\
z & double & Cartesian coordinates representation of RA and declination, as above. \\
flux & double & The flux measurements of a source, calculated from the mag value. \\
flux_err & double & The flux error of a source. \\
calmag & double & Calibrated mag. \\
flag & int & The source extraction uses a flag for a source to tell, for instance, if an object has been truncated at the edge of the image. \\
background & double & The source extraction estimates the background of the image. \\
threshold & double & The threshold indicates the level from which the source extraction should start treating pixels as if they were part of objects. \\
ellipticity & double & Ellipticity is how stretched the object is. \\
class_star & double & The source extractions classification of the objects. \\
\hline
\end{tabular}

\section{image}

time (jd), to which sky area this image belongs.

Images information includes observation skyarea

Each CCD may observe 2-3 sky areas, all in the manner of fixed point observations.
target
uniquecatalog

Accumulated source

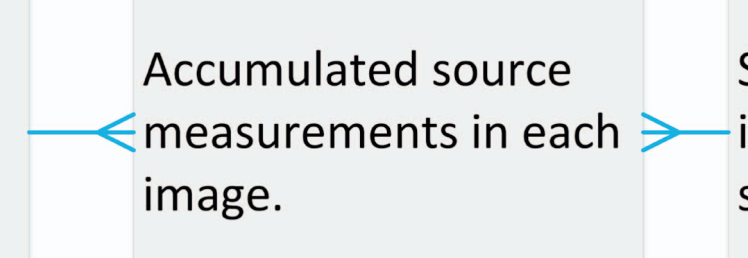

Sky-model, each entry is a unique identified source from targets.

Figure 3. Simplified database schema.

(A color version of this figure is available in the online journal.) 
inner relation uniquecatalog ordered by the predicate column "zone" to mimic a clustered index before the join phase:

\section{Algorithm 2.}

CREATE TABLE uO_zone AS SELECT * FROM uniquecatalog ORDER BY zone with data.

This has the advantage of scanning the outer relation sequentially. The time complexity of sorting the outer relation is $\mathcal{O}(n \log n)$, where $n$ is the size of the outer relation.

To reduce the amount of calculation and the space complexity, we use the standard SQL "WITH" clause to simplify complex SQL by materializing subqueries which save the MonetDB from recomputing multiple times. Although both the "WITH" clause and a temporary table can improve the query speed for complex subqueries, the former has some advantages over the latter: (1) "WITH" queries are treated as inline views without extra effort to remove the temporary tables after usage; and (2) WITH supports multiple subqueries and mutual references between subqueries. WITH allows us to assign a name to a subquery block. This name can be referenced in multiple places in the main query or even in the following WITH subquery. By using WITH clauses, we materialize the intermediate results target. "dec" - radius as decmin and target. "dec" + radius as decmax. The derived decmin/decmax are used in the next "WITH" subquery to compute the zone range: decmin/zoneHeight as zonemin and decmax/zoneHeight as zonemax. Zonemin and zonemax together define a zone range. The search radius can be flexibly changed according to the various different sky regions. The core SQL extract of the associates operator is listed below. Our test (Section 4.3.1) shows that the "WITH" optimization can bring the source association procedure for two tables with 175,597 rows $\times 175,540$ rows from resource exhaustion (running out of disk space) to $4 \mathrm{~m} 2 \mathrm{~s}$.

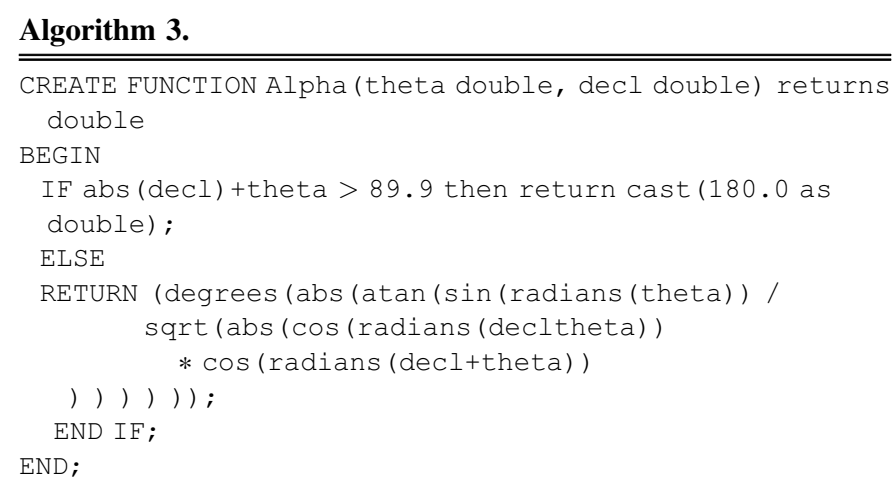

CREATE FUNCTION associates (imageno int, radius double) RETURNS TABLE (uniqueid bigint, targetid bigint, distance_arcsec double...) BEGIN

DECLARE TABLE u0_zone (LIKE uniquecatalog);

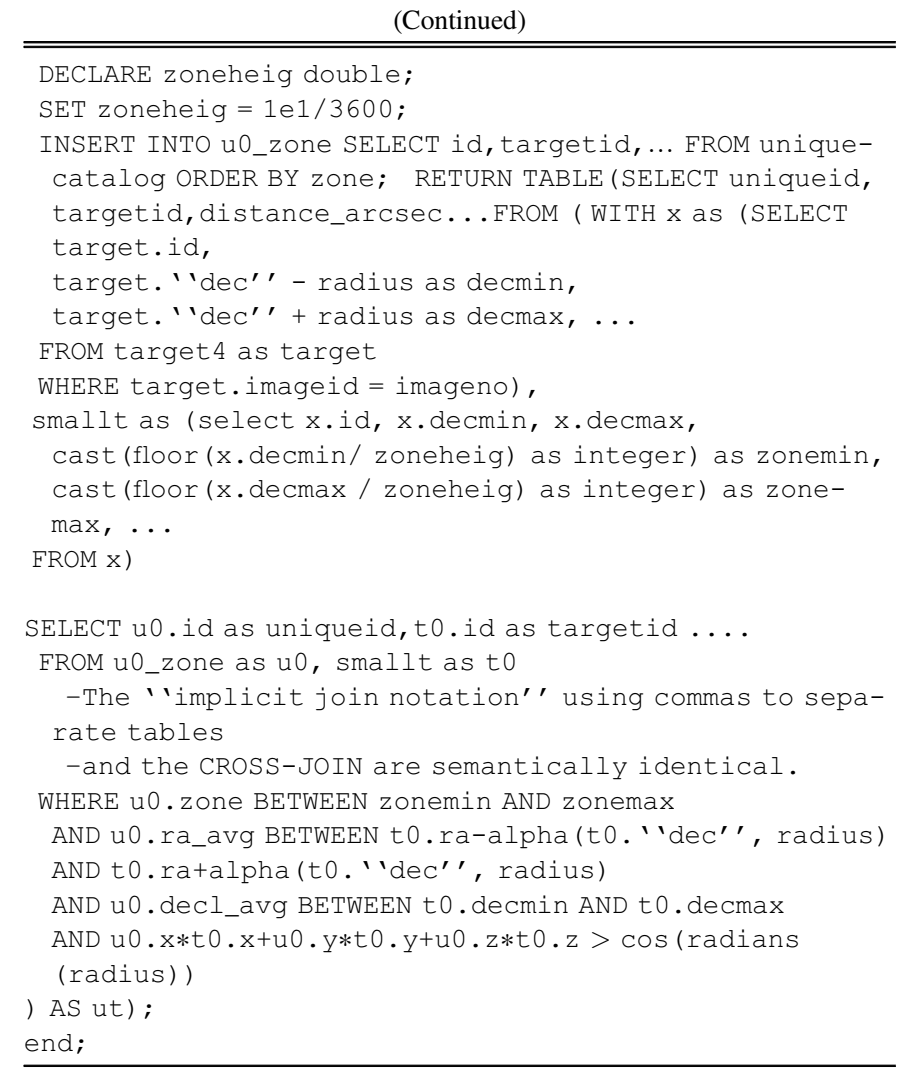

From inside the database engine, the optimization of the database implementation can speed up a RANGE-JOIN significantly. There are three expensive "range-join" predicates in the above associates function, i.e., the three BETWEEN... AND in WHERE clause. RANGE-JOINs are queries with inequality predicates (greater than, less than, or between) for which a column from the left table is restricted to be in a range specified by two columns of the right table. Because the condition with the highest discard rate is a RANGE-JOIN on the zone column, it should be given high priority in terms of optimization.

We have optimized the RANGE-JOIN implementation of the MonetDB by employing a quick binary search and compressed imprints index. The optimization has been introduced specifically for this work; however, it is generally applicable for all similar types of queries. The imprints index is already available in MonetDB but was not applied to RANGE-JOIN operations. This work has extended the imprints index to also work with RANGE-JOINs. Sidirourgos \& Kersten (2013) developed a novel cache-conscious secondary index structure called imprints to speed up scans over large tables stored in MonetDB. It is designed such that any clustering or partial ordering is naturally exploited without the need for extra 


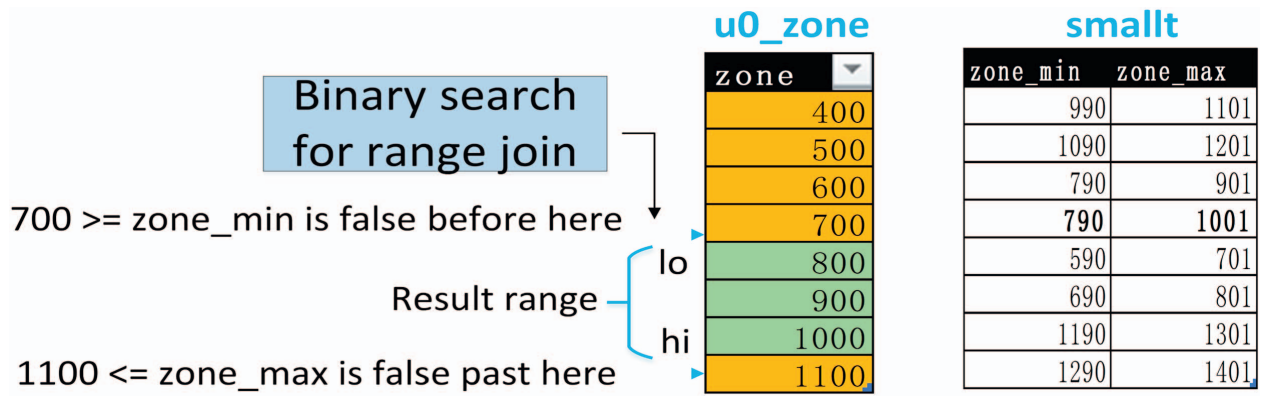

Figure 4. Optimized RANGE-JOIN in MonetDB.

parametrization. If the left column is sorted, then we use a binary search which allows for the majority of the table to be skipped to reduce join time by orders of magnitude instead of "brute-force" for large tables. Figure 4 illustrates an example of the binary search optimization. The table $u 0 \_z o n e$ with a column zone joins with the table smallt using a BETWEEN predicate (zone BETWEEN zone_min AND zone_max). When the zone range of the BETWEEN predicate is $(790,1001)$, only the tuples of zone values 800,900 , and 1000 (within the zone range) are quickly selected while all other tuples are skipped. If the left column is unsorted, then we use imprints under three conditions. (1) The data type is right for imprints. All numeric types (integers of all widths and floating points) and types that are internally represented as integers, such as dates and timestamps, are suitable for imprints. (2) The left column is either persistent or already has imprints. (3) The right column is long enough so that it is worth the effort of creating imprints. Without sorting the order on the ra_avg and decl_avg columns, the other two range-join predicates, i.e., $u 0 . r a \_a v g$ between $t 0$. ra-alpha(t0. "dec", radius) and t0.ra+alpha(t0. "dec", radius) and $u 0$.decl_avg between t0.decmin and t0.decmax, will use imprints to minimize data access.

The time complexity includes two parts: the complexity of sorting the left column is $\mathcal{O}(|l| \cdot \log |l|)$, and the complexity of the range join is

$$
T(|l|,|r|)= \begin{cases}|r| \cdot \log |l|, & \text { if } l_{\text {sorted }} \\ C \cdot|r| \cdot|l|, & \text { if } l_{\text {imprints }} \\ |r| \cdot|l| . & \text { if nested loop, }\end{cases}
$$

where $C$ is the size of the imprints of the left column. $C$ is $\ll 1$ in a typical case and $\frac{1}{16}$ in the worst case. Without optimization, a direct scan is employed using a nested loop, so $C=1$. As shown by our test, the optimization of the RANGE-JOIN implementation can reduce the source association procedure from $4 \mathrm{~m} 2 \mathrm{~s}$ further down to a few seconds, resulting in a speed-up by a factor of 220 (see Section 4.3.1 for the detailed performance testing).

\subsection{Optimization of One-to-many Match Type}

Figure 5 illustrates a simplified version of HCHDLP, which includes source associations and the subsequent processing of associated relationships in four types. Among these types, the one-to-many relationship is the slowest one. This relationship is caused by either a new image with higher spatial resolution or a newly detected source. When a one-to-many relationship occurs, the associatedsource table is traversed twice originally in $\mathrm{TraP}$ (Scheers 2011). The size of the associatedsource table is a function of the number of sources of the sky-model uniquecatalog table: $D_{\text {assoc }}=175600 \times 26 \times 2400 \times 36 \times n=367 \times n$ (GB), where $n$ is the number of observation days. For instance, at the epoch of image 5000, the associatedsource table has $1+$ billion rows. The EQUI-JOIN with the associatedsource table on a predicate of equal uniqueid takes $20.1 \mathrm{~s}$ to return 41,894 rows.

Since the EQUI-JOIN with associatedsource is so expensive, we have changed the strategy of forming the light curves of the one-to-many type to build the pipeline that can operate in real-time.

(1) We drop the uniqueness constraint by removing the primary key and foreign key on the large tables during the run time of HCHDLP. ${ }^{9}$ During idle time, the check is instead carried out by an SQL query, and the primary and foreign keys are added back to maintain the consistency of the database.

(2) In the one-to-many case, the old relationship between the uniquecatalog and the target table is replaced by a new one through "forking" (see Figure 6 for details). However, during the replacement, DELETE DML (deleting the old relationships from the big table associatedsource) is expensive. We avoid DELETE $^{10}$ by using INSERT to insert the old relationships into a small auxiliary table, uid_legacy. The Uid_legacy table stores deleted old uniqueids and their children (replacement)

\footnotetext{
9 Applicable to all of the match types of relationships.

${ }^{10}$ Frequent UPDATE and DELETE DML operations have been shown to be very expensive on a petabyte system (Becla et al. 2010), and so we tried to constantly apply the "write-once and never-update" and "append-only" principle to the big light curve table, which greatly optimizes HCHDLP throughput and latency.
} 


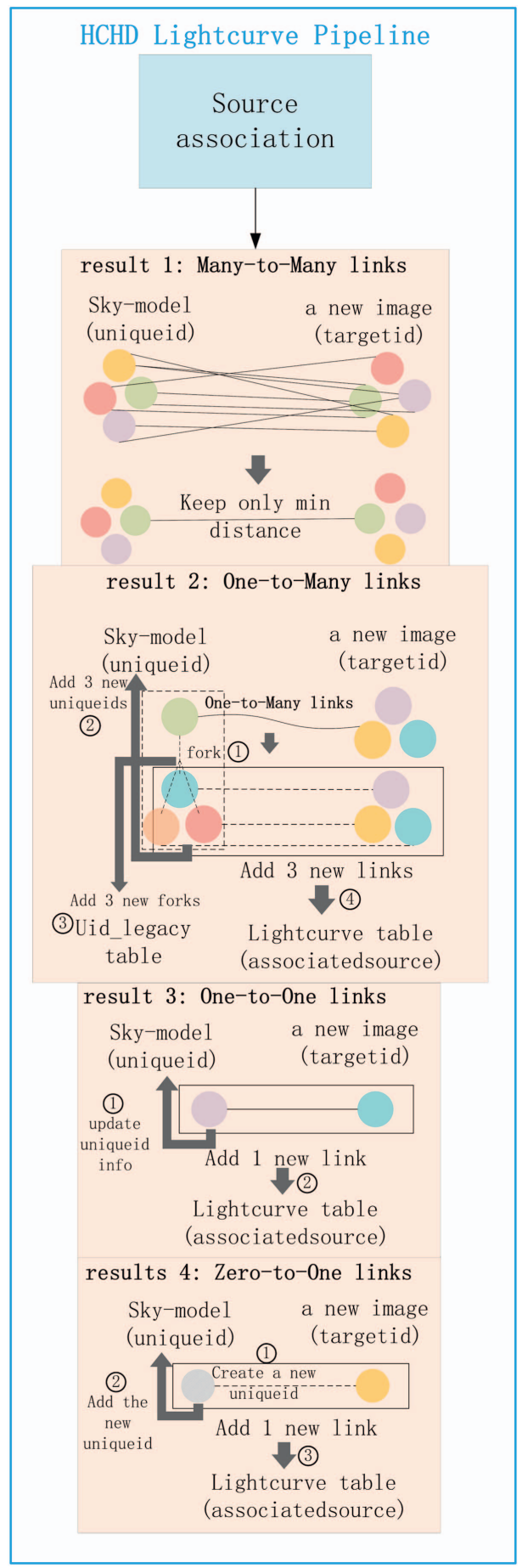

Figure 5. HCHDLP displays how to process four types of source association results. Many-to-many links are replaced with the link with minimal distance to reduce overhead. One-to-many links are replaced with many new links, which are inserted into the the light curve table. Old uniqueids and their successor relationships are inserted into the uid_legacy table. One-to-one links are previously found relationships. Zero-to-one links are newly detected targets which have no previously assigned uniqueid.

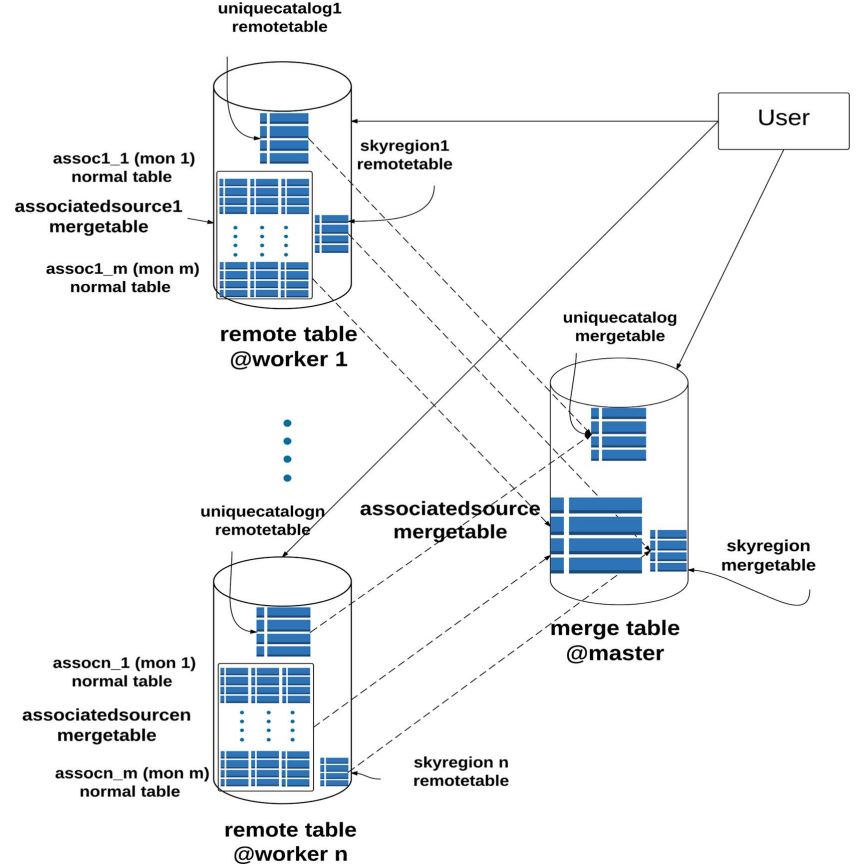

Figure 6. Distributed GWAC database architecture is comprised of one master node and multiple worker nodes. Remote tables associatedsource1..n, uniquecatalog $1 . . n$ on individual worker nodes are mapped to the merge table associatedsourceand uniquecatalog on the central server. Within a worker node, the associatedsource1..n table can be further partitioned by time, i.e., by months, weeks, or a couple of days. This time partitioning of the light curve table provides coarse temporal indexing and finer data locality, so that light curve queries involve only the relevant temporal partitions.

(A color version of this figure is available in the online journal.)

uniqueids in the one-to-many scenario and it will exist for all time as a historical record of the changes.

\section{Shared-nothing Distributed HCHDLP Database Architecture}

The catalog and light curve production of the temporary HCHDLP database will be periodically exported to the longterm distributed database. The shared-nothing distributed database is the best solution for storing the final products of HCHDLP over the long term thanks to independent hardware and software architecture. The architecture is adopted by taking into account the GWAC's top-level pointing strategy; there is no source association between the available sky regions of different CCDs, which is guaranteed by the GWAC's pointing strategy.

Figure 6 illustrates the shared-nothing distributed database architecture comprised of one master node and multiple worker nodes. The initial number of worker nodes should be the same as the number of CCDs. Each remote table on a worker node is 
mapped to the merge table with the same name as the master. In terms of the MERGE TABLE+REMOTE TABLE of MonetDB, the MERGE TABLE technique is a horizontal data partitioning method. The technique allows a table to be defined as the union of its partitions and enables finer control of data locality during query evaluation. As complements of the merge tables, the REMOTE TABLE technique allows the partitions of a merge table to reside on different nodes. Queries involving remote tables are automatically split into subqueries by the merge table and executed on the remote tables. The REMOTE TABLE adopts a straightforward master-worker architecture: one can place the partition tables in different databases and then concatenate everything together in a MERGE TABLE in the master database. In addition to REMOTE TABLEs on another node, local normal tables can also be added to be part of a MERGE TABLE. The MERGE TABLE supports nested merge tables, i.e., a MERGE TABLE can also contain other MERGE TABLEs. The MERGE TABLE and its partitions can be queried both individually and jointly.

In order to improve the performance of data querying, a remote table within a worker node can be further partitioned according to the ratio of the real RAM size and the data product size. The time span of each partition ranges from a couple of days to months.

There are a few sources that fall outside the boundaries of the observation areas, but the marginal sources are few. First, according to the share-nothing design, the sources of one CCD camera will not be associated with those of other cameras. Second, the GWAC survey strategy and its pointing and tracking system can ensure that each CCD observes a few fixed sky areas in the long term and that the drift of the pointing center is controlled within 10 pixels. Therefore, if a marginal source is detected in the area of a CCD, then it will be added to its catalog, and if the source moves out, then it might appear in another area and will be added to another catalog and traced. It will be flagged in the flag attribute of the sources catalog (see Table 1).

\section{Experiments and Evaluations}

This section describes our tests on the performance of both HCHDLP and the shared-nothing distributed database, including source association optimization on MonetDB and its comparison with PostgreSQL, HCHDLP running, and light curve queries.

\subsection{Hardware and Software Configuration for HCHDLP}

Our experiments run on a cluster of six server nodes of the CWI Scilens cluster platform interconnected via InfiniBand 40 $\mathrm{Gb} / \mathrm{s}$ links. Each node has two sockets, 32 hyper-threaded cores that use Intel Xeon CPU E5-2650 v2 @ 2.60 GHz, with $256 \mathrm{~GB}$ main memory and $5.4 \mathrm{~TB}$ of storage on software RAID0 containing three disk drives per server. Tests are conducted on the MonetDB default (development) branch v11.22.0, hg id 3603a1af9790. Optimized compilation of MonetDB is activated in our tests.

\subsection{Data and Loading}

A hindrance for the large-scale adoption of DBMSs in handling astronomical catalogs is the time it takes to bulk-load the data into the databases. Our experiments are based on the target table. It is ingested by simulated catalog files, whose sizes are proportional to the observation time. Simulated catalog files represent pseudo-sources extracted after pipeline 0 . The catalog files are synthesized by adding positions and flux noises to a template catalog file extracted from the UCAC4 catalog (Zacharias et al. 2013). Typically, each image is set to have $\sim 175,600$ sources, and each source has 22 column attributes, which means that an increment of thetarget table is $\sim 79 \mathrm{~GB}$ per night (with a cadence of $15 \mathrm{~s}$ and $10 \mathrm{~h}$ of observation per night) per CCD. We produce simulated catalog files in batches and load them into the target table before the HCHDLP system starts testing.

Binary bulk loading (MonetDB 2016) is used to load simulated catalogs into MonetDB. The SQL COPY command can take a complete ASCII file and insert the data in one go using all of the system cores in parallel. Furthermore, MonetDB created a binary bulk loading method, that is, the binary version of the COPY command: COPY BINARY. When large tables need to be loaded into a database, the binary bulk loading is slightly faster. This saves the rendering of data into ASCII and subsequent parsing of the data being exchanged and "attach" it to the SQL table. To illustrate this point, the SQL query below is used to load the binary column files into the target table.

\section{Algorithm 4.}

COPY BINARY INTO target FROM ('path_to_column_file_i'', ..., 'path_to_file_f'').

Each attachment file is produced using our simulator program that writes the binary version of the column files directly into the disk. All of the binary column files are aligned, i.e., the $i$ th value in each file corresponds to the $i$ th record in the table. The files with numerical data are moved into place to avoid copying (MonetDB 2016).

\subsection{Performance of HCHDLP}

\subsubsection{Source Association}

At the beginning, with the outside database optimization (i.e., sorted zone and "WITH" clause), the execution time of the source association for two tables of $\sim 175,600$ rows is as short as $4 \mathrm{~m} 2 \mathrm{~s}^{11}$ Then, the inside database optimization further reduces the time of the source association from $4 \mathrm{~m} 2 \mathrm{~s}$ to $1.1 \mathrm{~s}$ with a 220x speed up. The association radius is set to be 6

\footnotetext{
${ }^{11}$ Without the optimization, the source association failed in the same environment due to a huge crossproduct running out of disk space, and succeeded in a more powerful environment with $20 \mathrm{~TB}$ disk space in $59 \mathrm{~m}$.
} 
arcsec, that is, the same as the zone height which is a parameter in the zone algorithm. ${ }^{12}$ Since the highest discarding rate comes from RANGE-JOIN on the zone column, the 220x speed up is mostly due to the binary search on the sorted zone column. The following is the source association query by calling the Q26: associates function.

Algorithm 5.

\begin{tabular}{l}
\hline \hline sql> INSERT INTO tempuniquecatalog SELECT * FROM \\
associates $(2,0.00166666666666666666) ;$ \\
179,769 affected rows $(1.1 \mathrm{~s})$
\end{tabular}

The accuracy validation of our optimization is performed by both a self-join and cross-join of the source association and by checking if the correct number of new sources could be found. For a self-join with a radius of 0.36 arcsec, the sources in a simulated catalog with 175,597 rows all match themselves. The related code is listed as follows.

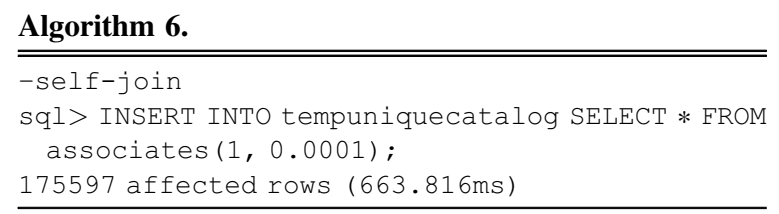

For a cross-join, the sources in two simulated catalogs with 175,597 rows $\times 175,540$ rows are cross-matched with a radius of 5 arcsec. The cross-join returns 27 unmatched sources because their distance from other sources are above 40 arcsecs. Again, the related code is listed as follows.

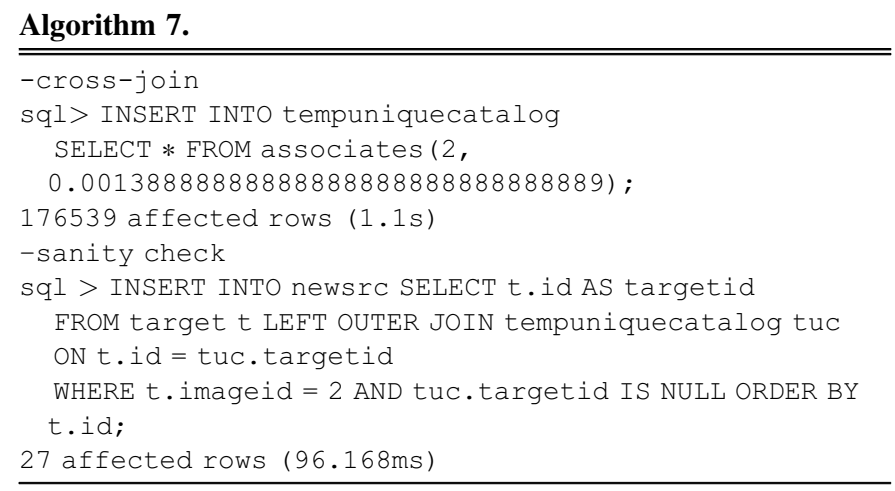

\footnotetext{
12 This association radius here is set to be half a pixel (11.7 arcsecond/pixel). The uncertainty of the object position is 0.1 pixel for our simulated catalogs. We employ 0.5 pixel $(5 \sigma)$ as the tolerance radius here, which is, in practice, consistent with our above PSF method of the tolerance radius selection (see Section 2).
}

\subsubsection{Comparison with PostgreSQL}

The performance of the source association based on the column-store database (MonetDB) is compared with a traditional row-store database (PostgreSQL) involving PostGIS. PostGIS $^{13}$ is an extension to PostgreSQL which allows it to handle and process geographic data through GiST-based R-Tree spatial indices. The GiST (Generalized Search Tree) index is the most suitable for querying spatial data. GiST is used to speed up searches on all kinds of irregular data structures (integer arrays, spectral data, etc.) which are not amenable to normal B-Tree indexing (Hellerstein et al. 1995). Although PostgreSQL supports three kinds of indices by default, i.e., B-Tree, R-Tree, and GiST, we adopt the GiST index in the current study. At first, B-trees are hard to deal with when applied to a two-dimensional sky image since they were originally designed for one-dimensional, linearly ordered key spaces. Second, R-Tree implementation is not as robust as GiST. For example, GIS objects larger than $8 \mathrm{~K}$ will cause the building of the R-Tree index to fail. Finally, the GiST index can support the nearest-neighbor search over large data sets, which is close to the purpose of source association (Kornacker 1999). The queries run on PostgreSQL are identical, with modulo syntax differences. The code below shows the experimental procedures using PostgreSQL.

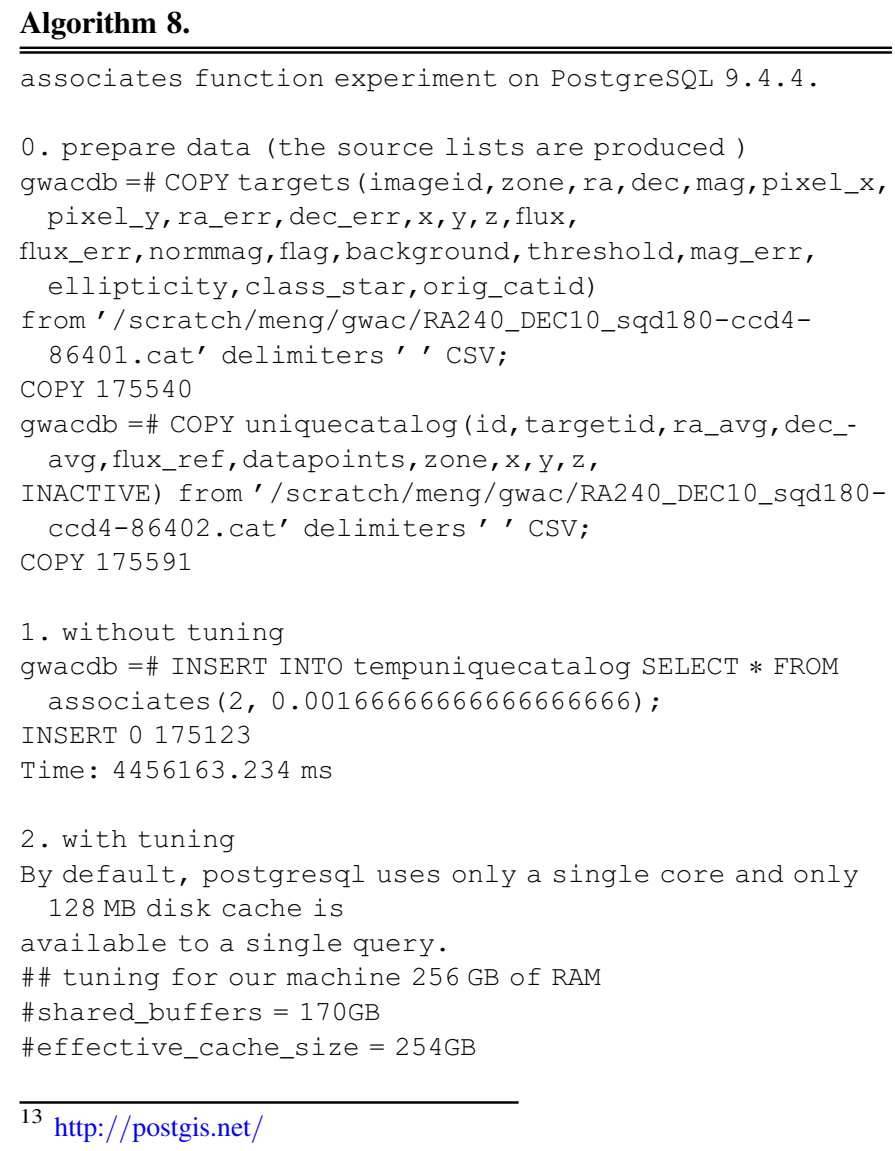


Table 2

Source Association Query Speed Comparison between MonetDB and PostgreSQL

\begin{tabular}{lcc}
\hline \hline Source Association Queries & Mean time (second) \\
\hline MonetDB with "WITH" clause & 242 \\
MonetDB with Range-Join optimized & 1.1 & \\
PostgreSQL & 4456 & \\
PostgreSQL tuned & 3074 & \\
PostgreSQL tuned and with GiST index & create index & 10.08 \\
& query & 3.89 \\
& sum & 13.97
\end{tabular}

Note. Both under optimization or indexing, MonetDB is $3.54 \mathrm{x}$ faster than PostgreSQL, even though extra time for creating a GiST Index is not taken into account in PostgreSQL. It is important to note that the GiST Index will be updated every time new data is inserted, so that data loading performance will become slower, and hence this will significantly delay the whole HCHDLP.

(Continued)

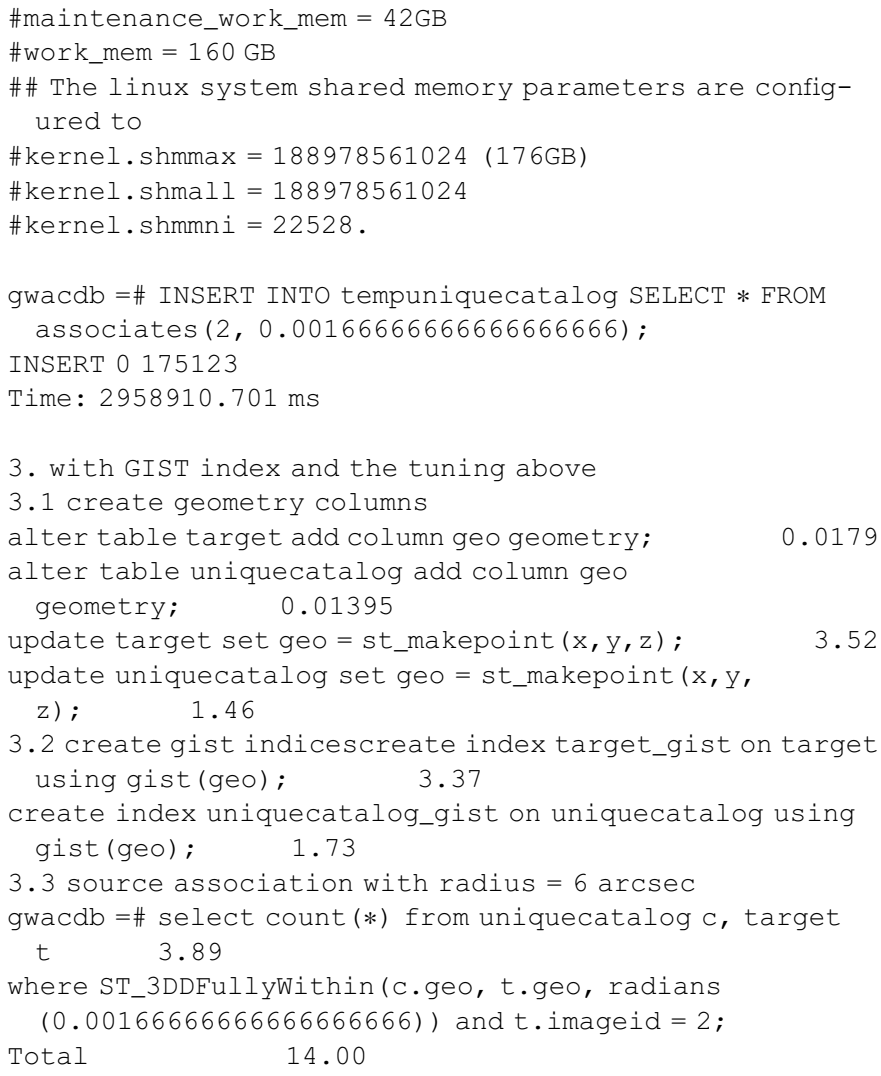

Table 2 compares the runtimes of source association in MonetDB and in PostgreSQL. All of the tests are based on the cross-match of two tables with 175,597 and 175,540 rows. The tuning parameters for the PostgreSQL database server are shared_buffers $=170 \mathrm{~GB}$, effective_cache_size $=254 \mathrm{~GB}$, and work_mem $=160 \mathrm{~GB}$. The linux system shared memory parameters for PostgreSQL are kernel.shmmax = kernel. shmall $=188978561024(176 \mathrm{~GB})$ and kernel.shmmni $=22528$ $(B)$. Note that only one process per database session can be utilized by PostgreSQL, and so a single complex and CPUintensive query is unable to use more than one CPU. In order to perform a fairer comparison to PostgreSQL, a single-core performance test of MonetDB is conducted by setting "sequential_pipe" as the SQL optimizer pipeline. The sequential_pipe allows the mserver5 to avoid using parallelism. The average run time is $1.7 \mathrm{~s}$. The single-core performance shows that MonetDB is faster than PostgreSQL, not only due to MonetDB's ability to harness multiple cores, but mainly due to the Range-Join optimization we applied to it.

By comparison, MonetDB (1.1s) is 3.54x faster than PostgreSQL (3.89s) even though the time to create an index in PostgreSQL is not included. It is worth noting that the GiST index needs to be updated when new data are inserted every 15 s (the cadence of GWAC). Such a frequent update causes the index building and data loading to become slow, which finally significantly degrades the pipeline performance.

\subsubsection{Performance of HCHDLP}

In principle, the sky-model uniquecatalog table grows over time due to new detections of events, e.g., optical transients, asteroids, and artificial objects, which will increase the execution time of HCHDLP. In order to test how the HCHDLP will perform in an extreme case, we carry out stress tests on HCHDLP by increasing the uniquecatalog table size through an enhanced artificial mismatch rate. The artificial mismatch is created by displacing the position of each source in the uniquecatalog through a random offset with a Gaussian distribution to produce simulated catalogs. Therefore, an extremely high number $(\sim 13,000)$ of new simulated sources is added to the uniquecatalog per day. Taking into account optical transients or other moving objects, all noise, and false positives, the upper limit of the new objects of GWAC is less than 1000 per day. Since the HCHDLP's main scientific goal is to manage the light curves of known objects, in practice, we will clean all of the above types of sources out of the uniquecatalog table every week. Hence, there will be very few new sources per day. During the 10 years of operation of GWAC, the HCHDLP performance should be stable.

Figure 7 shows the running time of HCHDLP and the increase of the light curve data size per day as a function of time over a period of $19 \mathrm{~d}$. Each point in the figure represents the time taken by the pipeline to produce the light curve data for the sources $(2400 \times 175600=0.42$ billion, $\approx 79 G B)$ observed in each night. Nineteen days of observations resulted in a target table of 8 billion rows (1.5 TB) and a light curve associatedsource table of 12.7 billion rows (318 GB). The 
19 days' lightcurve pipeline performance and everyday's lightcurve data volume

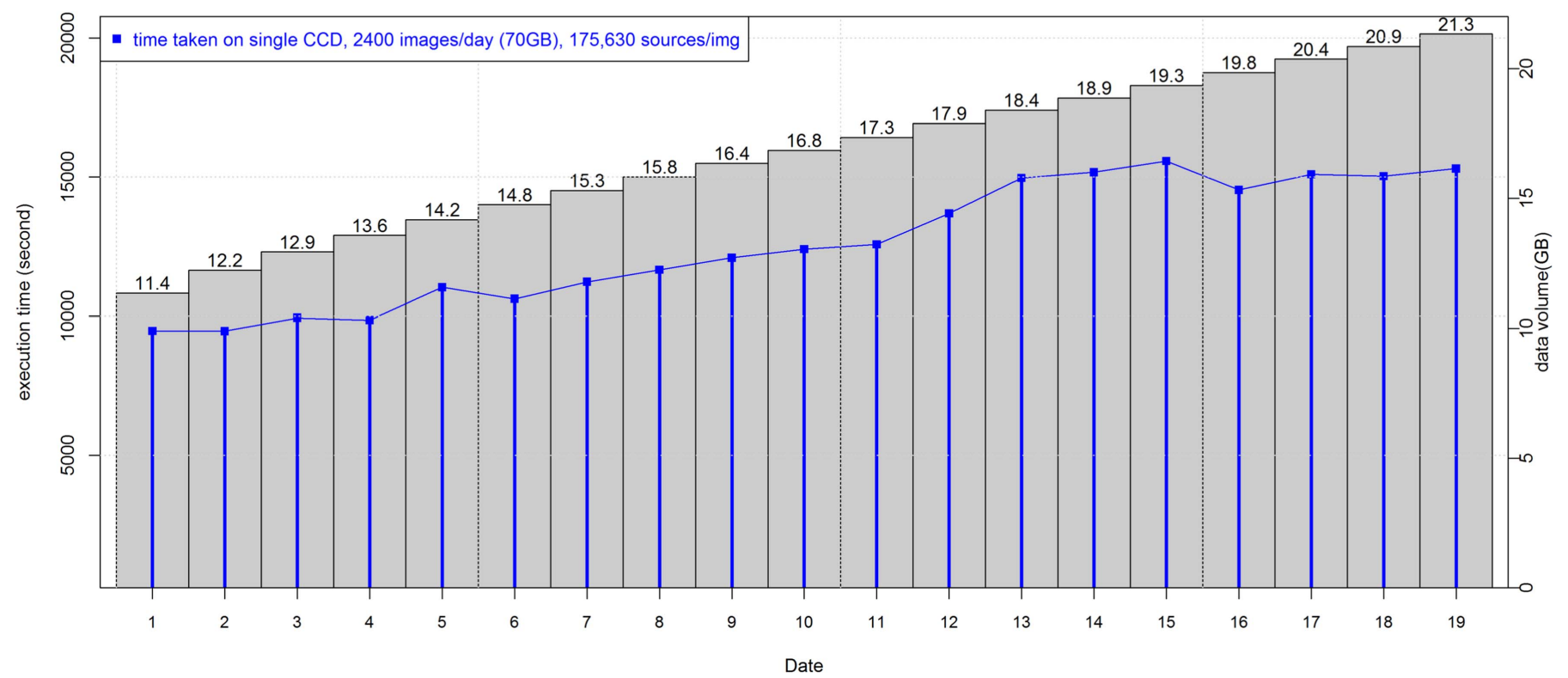

Figure 7. Light curve pipeline performance and the light curve data generated every day over a period of $19 \mathrm{~d}$; the execution time of the stress test goes up linearly with every day's increasing data size. For the first day's data volume, which is equivalent to GWAC daily data volume, the average time to process one image is 3.95 $\mathrm{s}$, which is much faster than the speed of GWAC's high-cadence image generation of $15 \mathrm{~s}$.

(A color version of this figure is available in the online journal.)

scripts we used to arrive at the results of this section are available on Github. ${ }^{14}$

We argue that the HCHDLP performance is acceptable in the context of GWAC based on our stress tests. On one hand, the execution time is roughly linear with the increase of light curve data per day, which is implied by the fact that both execution time and data volume increase with date in parallel (see Figure 7). On the other hand, the maximum execution time to process one image is derived to be less than $7 \mathrm{~s}$ from Figure 7, which is less than the GWAC cadence of $15 \mathrm{~s}$.

\subsection{Performance of Shared-nothing Distributed Database}

\subsubsection{Light Curve Retrieval on Partitioned Tables}

If the data volume of the light curve table associatedsource increases continuously night by night, then queries on the huge table would be very slow. However, astronomers are usually interested in data within certain time slices, and so in many cases, the results of a query can be achieved by accessing a temporal subset of partitions rather than the entire table-a technique that is called partition pruning. Partition pruning splits a large table into smaller, individual tables, and so

\footnotetext{
${ }^{14}$ https://github.com/wan-meng/gwac_pipeline, https://github.com/wanmeng/lightcurve-chunksize, https://github.com/wan-meng/concurrency
}

queries that access only a fraction of the data can run faster because there is less data to scan. Partition pruning dramatically reduces the amount of data retrieved from a disk and shortens the processing time, thus improving query performance and optimizing resource utilization (Herodotou et al. 2011).

In terms of the number of partitions, a larger number of smaller partitions provides finer granularity and causes less $\mathrm{I} / \mathrm{O}$ but also increases the management overheads. The more partitions we generate, the more partitions we have to deal with. Considering these trade-offs, we need to test which chunk size can provide the best response time for our testing system. In the test to determine the optimal chunk size for the associatedsource table, it is horizontally partitioned on ranges of ROW_NUMBER. Four partitioning ranges, i.e., chunk sizes, are tested to find which provides the best response time. Each chunk is created by accumulating the ROW_NUMBER of the associatedsource table to reach the size of $6 \times 10^{9}$, $12 \times 10^{9}, 24 \times 10^{9}$, and $48 \times 10^{9}$ rows, respectively. The SQL extract below is used to generate the timing information of Figure 8.

For each chunk size, the above queries of light curve retrieval are launched to search for all of the time series of flux measurements for a source of uniqueid $=1$ from all partitioning chunks, so that the WHERE clause does not specify which partitions are relevant for the query. 


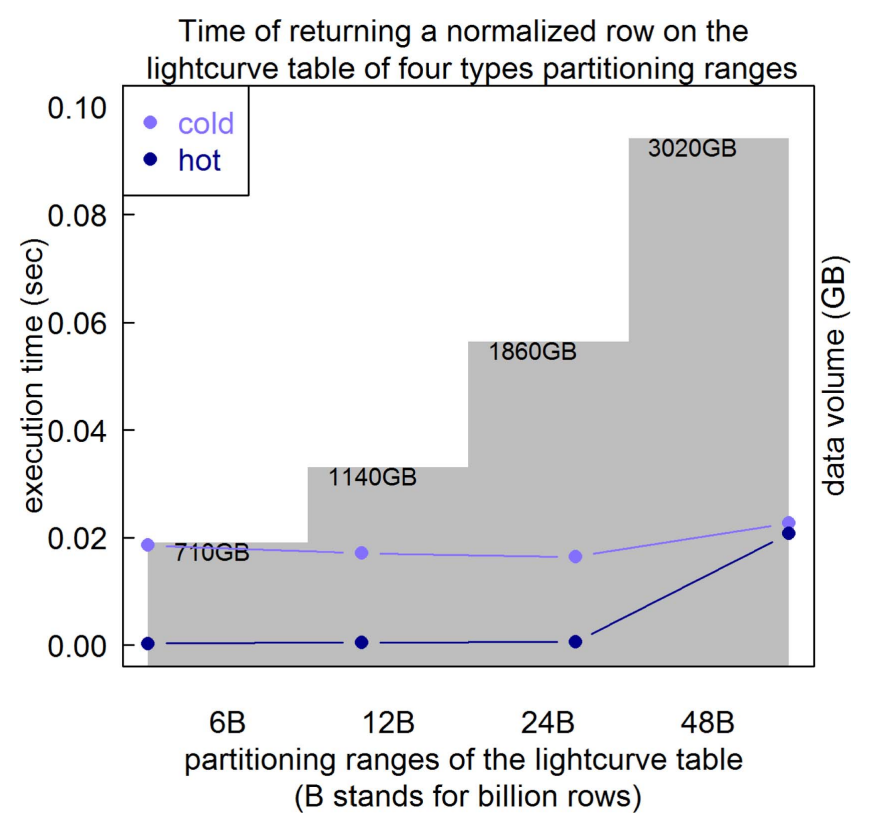

Figure 8. Performance of light curve retrieval over different partitioning chunk sizes.

(A color version of this figure is available in the online journal.)

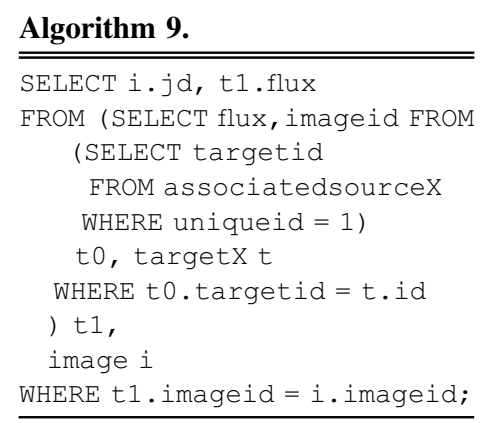

The response times are measured for both hot and cold runs. The hot runs refer to all of the necessary data which is already loaded into the main memory, while the cold ones are where data needs to be loaded into the memory before the queries start running. MonetDB is a main-memory database, the entire main memory of which can be viewed as a cache for disk I/O access (Manegold et al. 2002). MonetDB aggressively uses as much memory as is available, as many cores as possible in parallel without many tuning knobs, and tries to avoid going to a slow disk, so that caching effects have a significant impact on performance.

For chunk sizes of $6 \mathrm{~B}$ to $48 \mathrm{~B}$, the tested data size ranges from $710 \mathrm{~GB}$ for a period of $8 \mathrm{~d}$ to $3 \mathrm{~TB}$ for a period of $26 \mathrm{~d}$. The data set includes the light curve table associatedsource and the target table loaded into MonetDB beforehand. Then, we ran the above light curve retrieval query using uniqueid $=1$ and measured the (wall clock) time it took to produce the result. In cold runs, the database is stopped and all file system caches are emptied. In hot runs, we start up the database and run the query twice to warm up the caches. For both cold and hot runs, every query was run five times to control for random fluctuations in the system I/O and background activity.

Figure 8 shows the normalized response times of returning a row as a function of partitioning chunk size. It is obvious that the response time of the hot runs is much shorter than that of the cold runs, as expected. As we can see, for all of the cold runs, response times are almost unvaried. For the hot runs and chunk size between 6B and 24B, the MonetDB maps all needed data into the memory via the disk cache to greatly increase the performance by avoiding disk $\mathrm{I} / \mathrm{O}$. When the chunk size grows to $48 \mathrm{~B}$, related columns of the associatedsource table and target table touched by this query add up to $1035 \mathrm{~GB}$, that is, approximately four times the RAM capacity of $256 \mathrm{~GB}$. Therefore, the kernel swaps data out to free up some memory, which causes severe performance degradation. Therefore, our tests suggest that the chunk size of 24B is an optimal trade-off for our testing system. It is a good balance between the partitioning size of tables and performance.

\subsubsection{Scalable Concurrency}

Good concurrency is highly desirable. A database with good concurrency allows for a large number of users to access a database without any noticeable impact on performance (Bernstein \& Goodman 1981). Appropriate concurrent query execution facilitates improved resource utilization and aggregate throughput, while too much concurrency makes it a challenge to overall query performance (Duggan et al. 2011).

To provide both the fastest speed and highest concurrency of our database, the goal of this experiment is to find at which degree of concurrency the light curve retrieval query is fastest. In the experiment, we increase the number of concurrent users and measure the response time of random light curve retrievals on the distributed database, which is accessed either via the REMOTE table on the master node or via the local table on the worker nodes. We fire up $(1 / 10 / 15 / 20)$ parallel queries at a time to represent multiple users accessing the database with data volumes of $1 \mathrm{~TB}$ and $2 \mathrm{~TB}$. The test results are shown in Figure 9. In this figure, it is obvious that the response time of concurrent queries for $1 \mathrm{~TB}$ is much shorter than that for $2 \mathrm{~TB}$. In the $1 \mathrm{~TB}$ case, the average response time of a single query is $0.8 / 0.117 / 0.121 / 0.199$ for a concurrency of $1 / 10 / 15 / 20$. So, if we fire up $10 / 15 / 20$ queries at a time every $10 \mathrm{~s}$, then 60 queries will take a total of 7.02/7.28/11.94 $\mathrm{s}$ instead of $48 \mathrm{~s}$ for 1 user. In $1 \mathrm{~min}$, we will get throughputs of 60/90/120 queries/min. This test shows that scheduling appropriate concurrency can boost performance and throughput by efficient use of hardware resources. If we emphasize response time performance over throughput as a scientific program, then there should be no more than 15 concurrent users. However, when 

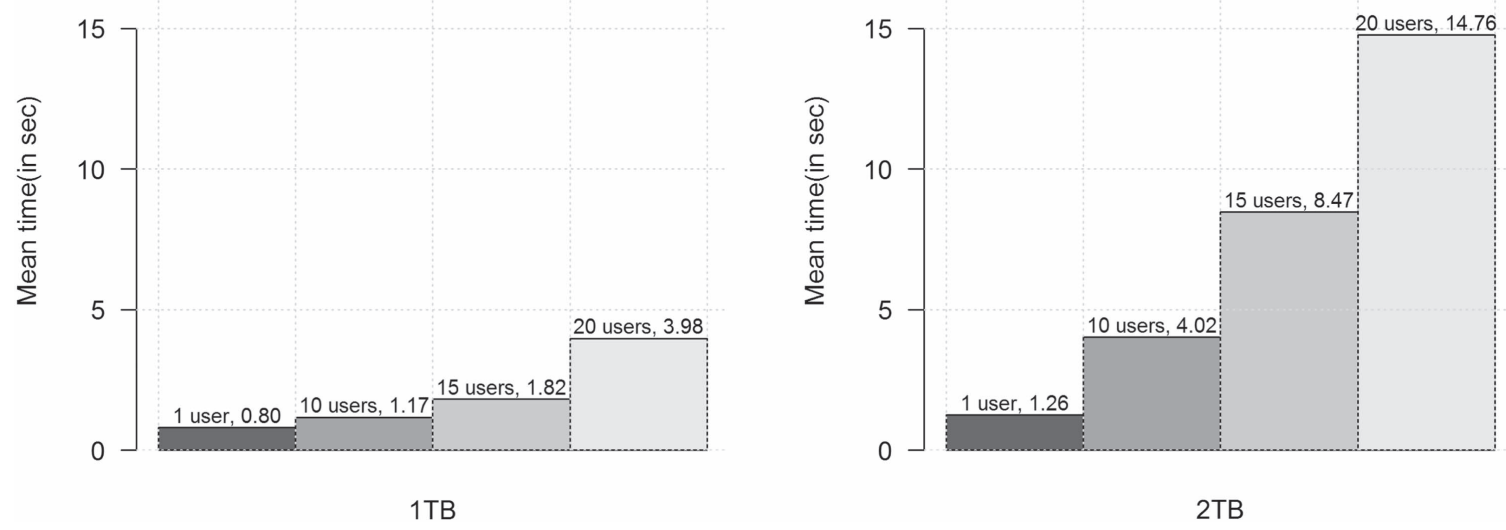

Figure 9. Single user vs. 10 vs. 20 users response time on $1 \mathrm{~TB} / 2 \mathrm{~TB}$ data sets.

more throughput is the evaluation criterion, 20 users is a better choice.

\section{Conclusion}

We develop a real-time light curve processing pipeline HCHDLP for the GWAC project. It is based on the columnstore database engine MonetDB and has been optimized for the large amounts of data of GWAC. From outside and inside the database engine, MonetDB has been improved to reduce the time consumed in the source association procedure. Outside the database engine, a sorted version of the inner relation ordered by the zone column is created before the join phase, and a SQL WITH clause is adopted to avoid multiple recomputations by MonetDB. Inside the database engine, the RANGE-JOIN query is implemented through a quick binary search and compressed column imprints. Furthermore, in building light curves of oneto-many match types, HCHDLP drops the uniqueness check during running and replaces DELETE with INSERT (i.e., insert the old relationships into a small auxiliary table).

As a result of optimization, our HCHDLP can process one catalog in $3.95 \mathrm{~s}$ on average, which is significantly shorter than the image cadence of $15 \mathrm{~s}$ of GWAC. Our tests show that the source association is sped up by a factor of $220 x$ relative to that before optimization. According to theoretical analysis, the time complexity is reduced from $\mathrm{O}(|r| \cdot|l|)$ to $|r| \cdot \log |l|$ (binary search) or $C \cdot|r| \cdot|l|$ (imprints, $C \ll 1$ ). We have also performed comparisons between MonetDB and PostgreSQL in performance of source association queries and find that the former takes $1.1 \mathrm{~s}$ on two tables with 175,597 rows $\times 175,540$ rows, which is $3.54 \times$ faster than the row-store disk-based database PostgreSQL.
We also built a shared-nothing distributed database to manage long-term light curves using a two-level time partitioning strategy via the MERGE TABLE and REMOTE TABLE technology of MonetDB. The optimal partitioning chunk size should provide both a short response time and fewer partitions, and is determined through tests. We find that the response time of MonetDB scales linearly with the number of users. In this scalable solution, both short- and long-running queries on large data sets are available which provide guidance for a solution to GWAC in the management of massive data.

In our future work, the HCHDLP may be further optimized in processing speed and match criteria. The GWAC real-time image processing must be completed in $15 \mathrm{~s}$ for one image, but the current implementation of pipeline 0 (see Figure 2) takes almost $10 \mathrm{~s}$ and HCHDLP takes $5.8 \mathrm{~s}$ (including both data loading and the cross-match). We note that pipeline 0 can be sped up through GPU implementation, and advanced algorithms based on our current optimization are available for the source association of HCHDLP. Moreover, the match criteria of source association will take into account the addition of a flux factor and the use of a variable tolerance radius with the brightness of objects to increase the accuracy of crossmatching. Our designed shared-nothing distributed database can provide one preliminary solution to managing light curves in the long term. It is certain that with advancing technology, there are other, possibly better, solutions that may overcome the data challenge. Additionally, there are some optimization options to reduce the light curve data volume, such as aggregate source characterization or reducing the time resolution of the data. Finally, estimating the scalability of HCHDLP performance over the full survey duration with realistic new source counts is key for a long-term astronomical project like GWAC. The upper limit of real source counts for GWAC is 
less than 1000 per day, taking into account optical transients or other moving objects, all noises, and false positives. In practice, we will clean all of the above kinds of sources out of the uniquecatalog table every week. Hence, new sources per day are very few and the HCHDLP performance over the survey duration should be stable in the long term.

The authors thank the anonymous referee for providing constructive comments which substantially helped to improve the quality of the paper. We also thank Prof. Yanxia Zhang, Dr. James E. Wicker, Dr. Xianmin Meng, Dr Xiaomeng Lu, Mr. Yang $\mathrm{Xu}$, Dr. Huali Li, Dr. Qichen Feng, and Dr. Lingjun Wang for their helpful discussions and modifications. This work was supported by a grant from the Sino-Dutch Scholarship Programme of the China Scholarship Council. It was also supported by the National Basic Research Program of China (973-program, grant No. 2014CB845800), and the National Natural Science Foundation of China (grant Nos. U1331202, 11533003, and U1431108).

\section{References}

Abadi, D., Boncz, P., Harizopoulos, S., et al. 2013, Found. Trends Databases, 5.3, 197

Banyer, J., Murphy, T. \& VAST Collaboration 2012, in ASP Conf. Ser. 461, Astronomical Data Analysis Software and Systems XXI, ed. P. Ballester, D. Egret, \& N. P. F. Lorente (San Francisco, CA: ASP), 725

Becla, J., Lim, K.-T., \& Wang, D. L. 2010, Data Sci. J., 8, MR1

Becla, J., Wang, D., Monkewitz, S., et al. 2013, LSST Database Design, http://1s.st/LDM-135

Becla, J., \& Wang, D. L. 2014, BAAS, 46, 303.03

Bernstein, P. A., \& Goodman, N. 1981, ACM Comput. Surv., 13, 185

Boncz, P. A., Manegold, S., Kersten, M. L., et al. 1999, in 25th International Conference on VLDB, ed. M. P. Atkinson et al. (San Francisco, CA: Morgan Kaufmann), 54

Burgett, W. S. 2012, Proc. SPIE, 8449, 84490T

Cordier, B., Wei, J., Atteia, J.-L., et al. 2015, arXiv:1512.03323

Dudik, R. P., Jordan, M. E., Dorland, B. N., et al. 2012, ApOpt, 51, 2877

Duggan, J., Cetintemel, U., Papaemmanouil, O., \& Upfal, E. 2011, in Proceedings of the 2011 ACM SIGMOD International Conference on Management of Data (Athens, Greece), 337

Gray, J., Liu, D. T., Nieto-Santisteban, M., et al. 2005, SIGMOD Rec., 34, 34
Gray, J., Nieto-Santisteban, M. A., \& Szalay, A. S. 2007, arXiv:cs/0701171 Gray, J., Szalay, A. S., Thakar, A. R., et al. 2004, arXiv:cs/0408031

Hellerstein, J. M., Naughton, J. F., \& Pfeffer, A. 1995, in Proceedings of the 21st International Conference on VLDB, ed. U. Dayal, P. M. D. Gray, \& S. Nishio (San Francisco, CA: Morgan Kaufmann), 562

Herodotou, H., Borisov, N., \& Babu, S. 2011, in 2011 ACM SIGMOD/PODS Conference (Athens, Greece), 49

Idreos, S., Groffen, F., Nes, N., et al. 2012, Bull. IEEE Comput. Soc. Tech. Comm. Data Eng., 35, 40

Idreos, S., Kersten, M. L., \& Manegold, S. 2007, in CIDR 2007: Third Biennial Conference on Innovative Data Systems Research (Online), 68

Ivezic, Z., Tyson, J. A., Abel, B., et al. 2008, arXiv:0805.2366

Jurić, M., Kantor, J., Lim, K. T., et al. 2015, arXiv:1512.07914

Kaiser, N., Aussel, H., Burke, B. E., et al. 2002, Proc. SPIE, 4836, 154

Koposov, S., \& Bartunov, O. 2006, in ASP Conf. Ser. 351, Astronomical Data Analysis Software and Systems XV, ed. C. Gabriel et al. (San Francisco, CA: ASP), 735

Kornacker, M. 1999, in Proceedings of the 25th International Conference on VLDB, ed. M. P. Atkinson et al. (San Francisco, CA: Morgan Kaufmann), 699

Manegold, S., Boncz, P., \& Kersten, M. L. 2002, in Proceedings of the 28th International Conference on VLDB (San Francisco, CA: Morgan Kaufmann), 191

Manegold, S., Kersten, M. L., \& Boncz, P. 2009, Proc. VLDB Endowment, 2,1648

MonetDB 2016, Binary Bulk Load, www.monetdb.org/Documentation/ Cookbooks/SQLrecipes/BinaryBulkLoad

Murphy, T., Chatterjee, S., Kaplan, D. L., et al. 2013, PASA, 30, e006

Norris, R. P. 2010, in Sixth IEEE International Conference on e-Science Workshops (Washington, DC: IEEE Computer Society), 21

Scheers, B. 2009, in ASP Conf. Ser. 411, Astronomical Analysis Software and Systems XVIII, ed. D. A. Bohlender, D. Durand, \& P. Dowler (San Francisco, CA: ASP), 143

Scheers, B. 2011, PhD thesis, Univ. Amsterdam

Scheers, B., Groffen \& TKP Team 2012, in ASP Conf. Ser. 461, Astronomical Data Analysis Software and Systems XXI, ed. P. Ballester, D. Egret, \& N. P. F. Lorente (San Francisco, CA: ASP), 757

Sidirourgos, L., \& Kersten, M. 2013, in 2013 ACM SIGMOD International Conference on Management of Data (New York), 893

Simmhan, Y., van Ingen, C., Heasley, J., \& Szalay, A. 2011, in Proceedings of the First Annual Workshop on High Performance Computing Meets Databases (Seattle, WA), 33

Swinbank, J. D., Staley, T. D., Molenaar, G. J., et al. 2015, A\&C, 11, 25

Van Haarlem, M. P., Wise, M. W., Gunst, A. W., et al. 2013, A\&A, 556, A2

Wang, D. L., Monkewitz, S. M., Lim, K.-T., \& Becla, J. 2011, in SC11 Conference: State of the Practice (Seattle, WA), 12

Zacharias, N., Finch, C. T., Girard, T. M., et al. 2013, AJ, 145, 44 